\title{
Analysis of thermionic bare tether operation regimes in passive mode
}

\author{
J. R. Sanmartín, ${ }^{1, a)}$ Xin Chen, ${ }^{2, b)}$ and G. Sánchez-Arriaga ${ }^{2, c)}$ \\ ${ }^{1}$ ETS de Ingeniería Aeronáutica y del Espacio, Universidad Politécnica de Madrid, Madrid 28040 , \\ Spain \\ ${ }^{2}$ Bioengineering and Aerospace Engineering Department, Universidad Carlos III de Madrid, Leganes, \\ Madrid 28911, Spain
}

(Received 25 October 2016; accepted 10 January 2017; published online 24 January 2017)

\begin{abstract}
A thermionic bare tether (TBT) is a long conductor coated with a low work-function material. In drag mode, a tether segment extending from anodic end $\mathrm{A}$ to a zero-bias point $\mathrm{B}$, with the standard Orbital-motion-limited current collection, is followed by a complex cathodic segment. In general, as bias becomes more negative in moving from $\mathrm{B}$ to cathodic end $\mathrm{C}$, one first finds space-chargelimited (SCL) emission covering up to some intermediate point $\mathrm{B}^{*}$, then full Richardson-Dushman (RD) emission reaching from $\mathrm{B}^{*}$ to end $\mathrm{C}$. An approximate analytical study, which combines the current and voltage profile equations with results from asymptotic studies of the Vlasov-Poisson system for emissive probes, is carried out to determine the parameter domain covering two limit regimes, which are effectively controlled by just two dimensionless parameters involving ambient plasma and TBT material properties. In one such limit regime, no point $\mathrm{B}^{*}$ is reached and thus no full RD emission develops. In an opposite regime, SCL segment BB* is too short to contribute significantly to the current balance. Published by AIP Publishing.

[http://dx.doi.org/10.1063/1.4974764]
\end{abstract}

\section{INTRODUCTION}

Electrodynamic-tether systems were originally made of a long insulated, but conducting, wire that was equipped with a big sphere as passive-anodic contactor and a hollow cathode or an electron gun as an active cathodic contactor. ${ }^{1-3}$ They have gone through three important transformations during the last quarter century, towards simplicity and reliability. The first one happened in 1993, when the bare tether concept was introduced. ${ }^{4}$ Instead of using big spherical collectors, the tether was left bare, without insulation, to collect electrons from ionospheric plasmas as a giant Langmuir probe. The second transformation did not involve tetherplasma current exchange but tether geometry. For equal length and mass, tape-like bare tethers collect current more efficiently than round tethers due to their larger perimeters. ${ }^{5}$ The tape-like geometry also improves notably tether reliability because it typically lowers well over one order of magnitude the probability of a tether-cut by small space debris. ${ }^{6}$ The third transformation brought the ED bare-tether concept to its full completion. If coated with a low work-function $(W)$ thermionic material, ${ }^{7,8}$ then the negatively biased (cathodic) tether segment can emit electrons at moderate temperatures as a giant emissive probe. This is called a thermionic bare tether (TBT), ${ }^{9}$ a propellant-less device that requires neither active cathode, such as hollow cathodes or electron guns, nor associated power or gas-feed subsystems.

Thermionic emitters had been mentioned for cathodic contact, along with hollow cathodes, in a pioneer work. ${ }^{4}$ The emergence of the TBT concept is now possible, thanks to the

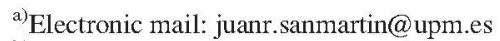

${ }^{b}$ Electronic mail: xin.chen $@ u c 3 m . e s$

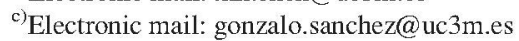

appearance of new materials with extremely low $W$ values and reasonably high temperature stability. A most promising material is the $\mathrm{C} 12 \mathrm{~A} 7: \mathrm{e}^{-}$electride, capable of reaching $W$ below $1 \mathrm{eV}^{7}$ With such a low work function, sufficient thermionic emission can be triggered merely by heating from the Sun. However, many aspects like the stability of this material under space conditions or the coating manufacturing process of a kilometers long tether remain open and will require further research work. It is of interest to develop theoretical models describing the complex current profile along a TBT cathodic segment, as a function of its $\mathrm{W}$ value, and to insert them in tether flight simulators to estimate tether performance, particularly in deorbiting spent rocket stages and spacecraft at end-of-life. TBT de-orbit efficiency need be compared against the standard bare tether that uses a hollow cathode for cathodic contact. ${ }^{10}$

The standard bare tether collects electrons over a segment that can nearly cover the entire tether. Tether efficiency, however, is greatly reduced if lacking cathodic contactor (and any active emission). Ions would arrive at the cathodic tether segment following the orbital-motionlimited (OML) law, each ion picking up an electron and leaving as neutral, electrons thus leaking out at the ion impact rate. Since ions are much heavier than electrons, the current exchange at negative bias is much smaller than the current collected at positive bias of equal magnitude. With a completely passive tether actually floating electrically, the anodic-to-cathodic length ratio and thus the length-averaged tether current are very small. Although unable to generate enough current for a sensible drag, floating bare tethers have been considered as upper atmosphere probes $^{11}$ and TBTs might represent a variant source of such artificial auroras. 
In the case of TBTs, its low-work-function coating makes thermionic emission significantly increase the cathodic current, thus improving efficiency. The operation of a TBT is as simple and reliable as drag-sail operation, except that characteristic Lorentz drag upon a TBT can be well over two orders of magnitudes higher than aerodynamic drag on a sail in Low Earth Orbit (LEO). The absence of active elements and the robustness of the underlying physics suggest that TBTs might be ideal devices for deorbit operations, which is a basic application of ED-tethers. ${ }^{12,13}$ It has recently been proposed to deorbit space debris from the Geostationary transfer orbits (GTO), where the Lorentz drag on a TBT, during each pass through an orbital arc around perigee, lowers the apogee progressively, making the object re-enter. ${ }^{14}$

Modeling TBT/ambient-plasma interaction is a challenging task that involves plasma physics of conventional and emissive Langmuir probes. Particle-in-cell simulations for planar and spherical emissive sheaths have been recently presented. ${ }^{15-17}$ The case of TBTs is different due to the cylindrical geometry. By using a preliminary model for the space-charge-limited (SCL) segment that incorporates the OML ion collection sheath ${ }^{5}$ and classical electron-sheath from an emitter, ${ }^{18}$ both current and potential profiles were first calculated without considering ohmic effects. ${ }^{9}$ The impact of ohmic effects was studied soon after. ${ }^{19}$

The present work contributes to the understanding of TBT-plasma interaction by organizing operational regimes in terms of two dimensionless parameters. It first introduces the basic TBT equations that govern bias and current profile along the tether (Sec. II). It is then shown that, even without a precise knowledge of the complex SCL emission law, it is possible to determine a parametric domain for which such emission can be ignored (Sec. III); also, in some approximate way, it is possible to determine a parametric domain for which just SCL emission takes place (Sec. IV). Conclusions are considered in Sec. V.

\section{BASIC TBT EQUATIONS}

Although TBT operation is passive and simple, its modelling is not. The tether-to-plasma bias, $\Delta V$, varies along the distance $s$ from the anodic end as

$$
\frac{\mathrm{d} \Delta V}{\mathrm{~d} s}=\frac{I(s)}{\sigma_{c} A_{t}}-E_{m},
$$

with $I(s)$ being the current intensity, $\sigma_{c}$ the tether conductivity, and $A_{t}$ the tether cross-sectional area. We have also introduced the projection of the motional electric field along the tether

$$
E_{m}=(\boldsymbol{v} \times \boldsymbol{B}) \cdot \boldsymbol{u}_{t},
$$

with $v$ the tether-to-plasma relative velocity, $\boldsymbol{B}$ the geomagnetic field, and $\boldsymbol{u}_{t}$ an unit vector along the straight tether in the direction of the current. The current along the tether, driven by this component of the motional electric field, results in a useful Lorentz force that acts in a passive mode as drag to de-orbit spacecraft.
Equation (1) holds over the entire tether length $L$, $0<s<L$, but the current on the right hand side (RHS) of this equation follows different laws at different segments. Because of the large disparity with tether radius, each point on the tether collects or emits current as if it were part of a long cylindrical probe uniformly polarized at the local bias. The sheath radius is of order of the Debye length of interest (typically fraction of centimeter in LEO) times the square root of the bias ratio ${ }^{5}$-highest at the anodic end - which keeps also very small as compared with the typical tether length that may be many kilometers long. The equation that governs the variation of the current intensity along the tether may be split, in principle, into three different tether segments (see Fig. 1).

$$
\frac{\mathrm{d} I(s)}{\mathrm{d} s}= \begin{cases}p_{t} \times j_{O M L}[\Delta V(s)] & 0<s<s_{B} \\ -p_{t} \times j_{S C L}[\Delta V(s)] & s_{B}<s<s_{B^{*}} \\ -p_{t} \times j_{R D}\left[T_{p}\right] & s_{B^{*}}<s<s_{C},\end{cases}
$$

where $p_{t}$ is tether perimeter, $j_{O M L}$ is the current density for the electron collection law, and $j_{S C L}$ and $j_{R D}$ are given by two different emission laws. These different laws can depend on the tether bias $\Delta V(s)$ or the tether temperature $T_{p}$.

As shown in Fig. 1, the TBT collects electrons from the anodic end $\mathrm{A}$ to a zero-bias point $\mathrm{B}$. Electron capture along this segment is modeled using the OML, maximum current law, assuming high bias $\left(e \Delta V \gg k T_{e}\right.$, where $T_{e}$ is electron temperature)

$$
j_{O M L}=\frac{e N_{\infty}}{\pi} \sqrt{\frac{2 e \Delta V}{m_{e}}},
$$

with $N_{\infty}$ the ambient plasma density, $e$ the electron charge, and $m_{e}$ the electron mass. ${ }^{5}$ Although high bias does not apply in a region very close to $\mathrm{B}$, the current collected in that region is negligibly small. Considering round tethers for simplicity, OML current collection was found to require probe radius less than some maximum radius $R_{\max }$ of order of the ambient Debye length (a corresponding condition for tapes requiring a maximum tape width, $w_{\max }=4 R_{\max }$ ). ${ }^{5}$

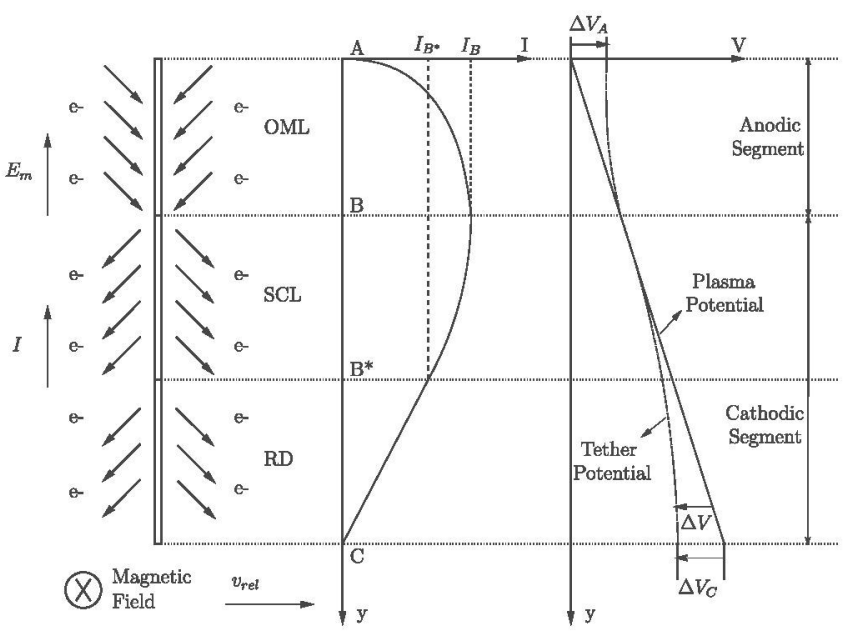

FIG. 1. TBT current and voltage profiles at the SCL-RD regime. 
Attracted electrons conserve both angular momentum $J$ and energy $E$, in terms of electrostatic potential and radial and azimuthal velocities

$$
\begin{gathered}
J=m_{e} r v_{\theta}=\text { const. } \\
E=\frac{1}{2} m_{e} v_{\theta}^{2}+\frac{1}{2} m_{e} v_{r}^{2}-e \Phi(r)=\text { const. }, \quad \Phi>0 .
\end{gathered}
$$

They also have decreasing $r$ and $v_{\theta}$ increasing in Eq. (4), requiring a net decrease from the last two terms in $\mathrm{Eq}$. (5), while keeping $v_{r}^{2}$ positive. As found in Ref. 5, such decrease requires a radial profile satisfying $r^{2} \Phi(r) / R^{2}>\Phi(R) \equiv \Delta V$ (with $\Phi$ increasing monotonically with decreasing $r$ ) and leads to the maximum $R$ condition.

As regards the cathodic segment, as tether bias becomes more negative in moving from $\mathrm{B}$ to the cathodic end $\mathrm{C}$, one first finds, in general, certain space-charge-limited (SCL) emission regime covering up to some intermediate point $\mathrm{B}$ *, then, possibly, Richardson-Dushman emission from $\mathrm{B} *$ to $\mathrm{C}$.

This splitting of the emission law, leading to two types of cathodic segments, arises from conditions that are, in a way, the opposite of those leading to the OML law holding between points $\mathrm{A}$ and $\mathrm{B}$. The energy equation for emitted electrons reads

$$
E=\frac{1}{2} m_{e} v_{\theta}^{2}+\frac{1}{2} m_{e} v_{r}^{2}+e\left(|\Phi(r)|-\left|\Phi_{p}\right|\right)=\text { const. }
$$

with $\Phi<0$. Here, the (emitted) electrons have increasing $r$ and thus decreasing $v_{\theta}$ in $\mathrm{Eq}$. (4), requiring a net increase from the last two terms in Eq. (6). In case $|\Phi(r)|$ decreases monotonically with increasing $r$, the overall result is continuous radial acceleration, no emitted electrons finding barrier in their way to infinity. At the Richardson-Dushman (RD) segment, with negative enough tether bias, the radial potential distribution is indeed monotonic.

The Richardson-Dushman emission law reads

$$
j_{R D}=A T_{p}^{2} \exp \left(-W / k T_{p}\right),
$$

with $A=4 \pi e m_{e} k^{2} / h^{3}=1.2 \times 10^{6} \mathrm{~A} \mathrm{~m}^{-2} \mathrm{~K}^{-2}$ a universal constant and $h$ the Planck constant. The work function $W$ for a given material, typically given in $\mathrm{eV}$ units, is the minimum thermodynamic work needed to help an electron inside the conductor to overcome the potential-energy barrier near the surface region, thus to be able to emit the electron from the surface. As a result, for a given material, the RD current is a function of the temperature. It can also be written in terms of a half-Maxwellian emitted-electron distribution, the density just at the probe thus related to $T_{p}$ and $W$

$$
j_{R D}=e N_{e m p} \times \sqrt{\frac{2 k T_{p}}{\pi m_{e}}}, \quad N_{e m p} \equiv \nu N_{\infty},
$$

where we introduced the dimensionless ratio $\nu$ of characteristic emitted and ambient electron densities. ${ }^{20}$

Considering an emissive probe at given negative bias $\Delta V$ and increasing emission parameter $\nu$, the effect of negative space charge near the probe will obviously increase. The same applies at given emission level $\nu$, when probe bias is made less negative. This condition does apply at the cathodic tether segment if moving in Fig. 1 upwards from end $\mathrm{C}$, at given ambient plasma conditions and emission parameters $W$ and $T_{p}$. The net effect is reducing the electric field at the tether, which at some point (the very point $\mathrm{B}^{*}$ ) will vanish. Moving up above $B^{*}$ - a potential dip in front of the tether sets in, the electric field pointing away from the tether, thus pushing emitted electrons, not energetic enough, back to it.

The effectively emitted current density in the segment $\mathrm{BB} *$ is thus smaller than the RD value, $j_{S C L}<j_{R D}$. A fully consistent and general description for $j_{S C L}$ is not available yet. It is written now for convenience as a fraction of $j_{R D}$

$$
j_{S C L}=f \times j_{R D}, \quad f<1,
$$

with $f$ satisfying conditions $f=1$ at $\mathrm{B} *$ and $f=0$ at $\mathrm{B}$.

Important uncertainties exist not only on models for factor $f$ in the current emitted within the SCL segment but also on the exact location of point $\mathrm{B} *$. However, because of the mentioned uncoupling of radial and longitudinal profiles due to $R$ and $L$ values disparity, the $\Delta V_{B^{*}}$ value in the profile does not require the emission law in the SCL segment. For cylindrical emissive probes in the high bias limit, and probe radius $R \approx R_{\max }$, ensuring collection of ions in the OML regime, an asymptotic analysis of the Vlasov-Poisson system can find self-consistently the radial sheath structure for a monotonic radial potential distribution with full RDemission. $^{20}$

For particular values of parameters

$$
T_{i}=T_{e} \equiv T=4 T_{p}=0.1 \mathrm{eV},
$$

i.e., a TBT temperature about $290 \mathrm{~K}$, a normalized electric field at the probe is shown in Fig. 2(a) versus probe bias for several emission levels. The dimensionless bias $e\left|\Delta V_{B^{*}}\right| / k T$ where that electric-field value rapidly decreases to zero is $26.2,89.7,143.0,238.8$, and 438.1 , for $\nu=20,50,70,100$, 150 , respectively, values fitting within a few percent a power law $^{20}$

$$
\frac{e\left|\Delta V_{B^{*}}\right|}{k T}=\left|\Delta \tilde{V}_{B^{*}}(\nu)\right| \approx 0.39 \nu^{1.4},
$$

as shown by the dashed curve in Fig. 2(b). This result will be used in both Sections III and IV for discussing operational regimes.

For a tether of a certain radius, immersed in a certain plasma environment, the tether may operate in different regimes, dependent on two normalized parameters, emission level $\nu$ and electromotive force ratio $e E_{m} L / k T_{e}$. If $e E_{m} L / k T_{e}$ is low enough and/or $\nu$ is high enough, the potential drop in plasma potential is reduced and the cathodic segment is unable to reach negative enough bias relative to the plasma to allow RD emission. In this regime, there is only SCL segment, with no sub-segment $\mathrm{B}^{*} \mathrm{C}$. On the contrary, if $e E_{m} L /$ $k T_{e}$ is high and/or $\nu$ is low, cathodic bias readily reaches the low value required for $\mathrm{RD}$ emission; in this regime, the subsegment $\mathrm{BB} *$ is very short and SCL current emission is negligible, thus requiring no SCL emission modeling, In a general regime, $\mathrm{SCL}$ and $\mathrm{RD}$ emissions are comparable. 

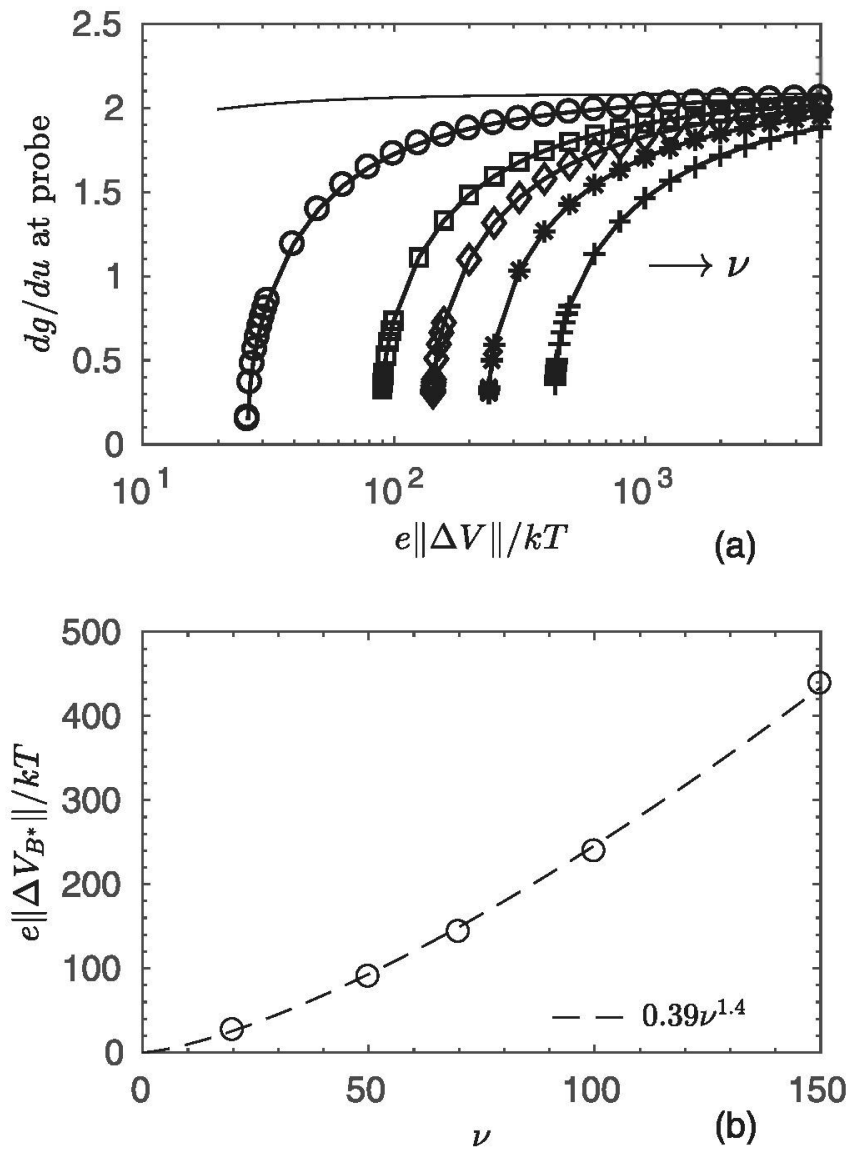

FIG. 2. For condition $R=R_{\max } \approx \lambda_{D}, T_{i}=T_{e} \equiv T=4 T_{p}=0.1 \mathrm{eV}$, (a) presents a normalized electric field at the probe (see Ref. 14) for the normalization definition) versus normalized probe bias at emission levels $\nu=0,20$, $50,70,100,150$; (b) presents the probe bias at which the electric field vanishes at the probe; the circles are calculated values for $\nu=20,50,70,100$, 150 and the dashed curve is the fitting law $0.39 \nu^{1.4}$.

\section{NEGLIGIBLE SCL-EMISSION REGIME}

Consider first the standard bare-tether analysis of coupled Eqs. (1) and (2), for the anodic segment $A B$, with the OML law in Eq. (3). For small values of the ratio

$$
\left(\frac{L}{L^{*}}\right)^{3 / 2} \equiv \frac{4}{3 \pi} \frac{p_{t} e N_{\infty} L}{\sigma_{c} E_{m} A_{t}} \sqrt{\frac{2 e E_{m} L}{m_{e}}}
$$

defining a length $L^{*}$ that gauges ohmic and current-collection impedances, ohmic effects will be small and current will be throughout a small fraction of the short circuit value, ${ }^{4} i \equiv I /$ $\sigma_{c} E_{m} A_{t}$. We then have

$$
\Delta V=E_{m}\left(s_{B}-s\right), \quad \mathrm{d} I / \mathrm{d} s \propto \sqrt{s_{B}-s},
$$

ignoring ohmic effects for simplicity. Straightforward integration of Eqs. (1) and (2), using conditions $I_{A}=0, \Delta V_{B}=0$, then yields a simple approximate relation between current and position at point $\mathrm{B}$

$$
i_{B} \approx \frac{1}{2}\left(\frac{L}{L^{*}}\right)^{3 / 2} \tilde{s}_{B}^{3 / 2} \ll 1
$$

where we introduced the dimensionless length $\tilde{s} \equiv s / L$.
Next, we consider Eq. (1) for the cathodic segment BB*. Integrating from $\mathrm{B}$ to $\mathrm{B}^{*}$ yields

$$
-\Delta V_{B^{*}}=E_{m}\left(1-\frac{I_{a v}}{\sigma_{c} E_{m} A_{t}}\right)\left(s_{B^{*}}-s_{B}\right),
$$

where the current average over the segment $\mathrm{BB}^{*}, I_{a v}$, is clearly less than $I_{B}$ and may thus be ignored in Eq. (14), which we rewrite as

$$
\tilde{s}_{B^{*}}-\tilde{s}_{B} \approx \frac{\left|\Delta V_{B^{*}}(\nu)\right|}{E_{m} L}=\frac{\left|\Delta \tilde{V}_{B^{*}}\right|}{e E_{m} L / k T} \approx \frac{0.39 \nu^{1.4}}{e E_{m} L / k T} .
$$

This shows the $\mathrm{BB}^{*}$ segment to be short within a relevant domain of operational parameters, such as the $\nu$ values in Fig. 2 and $E_{m} L$ values of many hundreds of volts; for $\nu=70$ and $e E_{m} L / k T=4500$, say, the ratio in Eq. (15) is about 0.033 .

Consider now current exchange in the full segment from $\mathrm{B}$ to C. Full RD emission applies from $\mathrm{B}^{*}$ to $\mathrm{C}$. The integration of Eq. (2) along $\mathrm{B} * \mathrm{C}$, with the boundary condition $I_{C}=0$, then yields

$$
I_{B^{*}}=p_{t}\left(L-s_{B^{*}}\right) j_{R D} .
$$

Similarly, integration of Eq. (2) from B to B* yields

$$
I_{B}-I_{B^{*}}=f_{a v} \times p_{t}\left(s_{B^{*}}-s_{B}\right) j_{R D},
$$

where, again, $f_{a v}$ is an average over the segment $\mathrm{BB} *$. Adding Eqs. (16) and (17), using Eq. (8) and dimensionless variables, then gives

$$
i_{B}=\frac{p_{t} e N_{\infty} L}{\sigma_{c} E_{m} A_{t}} \times \nu \sqrt{\frac{2 k T_{p}}{\pi m_{e}}} \times\left[1-\tilde{s}_{B^{*}}+\left(\tilde{s}_{B^{*}}-\tilde{s}_{B}\right) f_{\alpha v}\right] .
$$

Finally, equating $i_{B}$ values in Eqs. (18) and (13), using Eq. (11) yields

$$
\frac{3 \sqrt{\pi}}{4} \times \nu \sqrt{\frac{k T}{e E_{m} L}} \times\left[1-\tilde{s}_{B^{*}}+\left(\tilde{s}_{B^{*}}-\tilde{s}_{B}\right) f_{a v}\right] \approx \tilde{s}_{B}{ }^{3 / 2} .
$$

For representative values such as $\nu=70, e E_{m} L / k T=4500$, (a), the full factor multiplying the bracket on the LHS of Eq. (19) is of order unity. Then, Eq. (19), where, (b), $\tilde{s}_{B^{*}}-\tilde{s}$ is very small, does require $1-\tilde{s}_{B^{*}}$ to be of order unity. Otherwise, its LHS and RHS would be small and about one, respectively.

It follows from a ratio resulting from Eqs. (16) and (17):

$$
\frac{I_{B}-I_{B^{*}}}{I_{B^{*}}}=f_{a v} \frac{\widetilde{s}_{B^{*}}-\widetilde{s}_{B}}{1-\tilde{s}_{B^{*}}} \ll 1,
$$

that the current, in the regime above, varies very little over the segment $\mathrm{BB}^{*}$, thus making modeling of SCL emission unnecessary.

With the SCL segment negligible, the current profile is very simple. Following Eqs. (13) and (18) gives

$$
i(s) \approx \frac{1}{2}\left(\frac{L}{L^{*}}\right)^{3 / 2}\left[\tilde{s}_{B}^{3 / 2}-\left(\tilde{s}_{B}-\tilde{s}\right)^{3 / 2}\right], \quad \tilde{s}<\tilde{s}_{B},
$$




$$
i(s)=\frac{p_{t} e N_{\infty} L}{\sigma_{c} E_{m} A_{t}} \times \nu \sqrt{\frac{2 k T_{p}}{e E_{m} L}}(1-\tilde{s}), \quad \tilde{s}>\tilde{s}_{B},
$$

where $\widetilde{s}_{B}$ follows from Eq. (19):

$$
\frac{3 \sqrt{\pi}}{4} \times \nu \sqrt{\frac{k T}{e E_{m} L}}\left(1-\tilde{s}_{B}\right) \approx \tilde{s}_{B}^{3 / 2} .
$$

These results can be used to find simple analytical formulae for the Lorentz force and torque, which are useful to make preliminary mission design of deorbiting missions with TBTs.

\section{NO RD-EMISSION REGIME}

For $\nu$ increasing and/or $e E_{m} L / k T$ decreasing, both points (a) and (b) after Eq. (19) start failing. Consider then a case where $\mathrm{B}^{*}$ would lie just at tether end $\mathrm{C}$, i.e., $\tilde{s}_{B^{*}}=1$, $i_{B^{*}}=0$. Assuming that Eq. (10) still holds, Eqs. (19) and (15) read

$$
\begin{gathered}
\frac{3 \sqrt{\pi}}{4} \times \nu \sqrt{\frac{k T}{e E_{m} L}} \times\left(1-\tilde{s}_{B}\right) f_{a \nu} \approx \tilde{s}_{B}{ }^{3 / 2}, \\
1-\tilde{s}_{B} \approx \frac{0.39 \nu^{1.4}}{e E_{m} K / k T} .
\end{gathered}
$$

To determine $f_{a v}$, we model SCL emission from the cathodic segment $\mathrm{BB}^{*}$ as a double layer involving emitted electrons and attracted ambient ions (neglecting ion current and repelled ambient electrons) ${ }^{18}$ This is simplified by using the sheath radius in OML ion collection as the anodic radius in the classical analysis of emission in vacuum from an inner cylinder to a coaxial anodic one. ${ }^{9}$ In the no ohmic-effects limit, $\Delta V(s)=E_{m}\left(s_{B}-s\right)$, this leads to a linear profile

$$
f=\frac{s-s_{B}}{s_{B^{*}}-s_{B}}
$$

and $f_{a v}=0.5$.

Using Eqs. (24) and (25) to determine $\tilde{s}_{B}{ }^{3 / 2}$, and using this back in Eq. (25), determines a relation between parameters $\nu$ and $e E_{m} L / k T$,

$$
e E_{m} L / k T=0.39 \nu^{1.4}+0.41 \nu^{1.6}
$$

represented as a solid line in Fig. 3. To its right and below it, there is no segment $\mathrm{B}^{*} \mathrm{C}$ and no $\mathrm{RD}$ emission. The no $\mathrm{RD}$ transition occurs at $e E_{m} / k T$ values typically one order of magnitude greater than corresponding $\nu$ values. To $e E_{m} /$ $k T=3000$ value, marked in the figure, does correspond $\nu \approx 218.3$; the power approximation yields $\left|\Delta \tilde{V}_{B^{*}}(\nu)\right|$ $\approx 0.39 \nu^{1.4} \approx 732.7$, which is $6 \%$ smaller than the corresponding numerical value, 779.4 .

The dotted line represents that power approximation, which keeps below but close to that solid line at lower $\nu$ values. Moving up from the solid line, at constant $\nu$, RD emission starts at zero, but it would be expected to be a growing fraction of total (SCL $+\mathrm{RD}$ ) emission, becoming dominant at $e E_{m} L / k T$ values well above the corresponding value in the dotted line, making the contribution of the $\mathrm{BB}^{*}$ segment

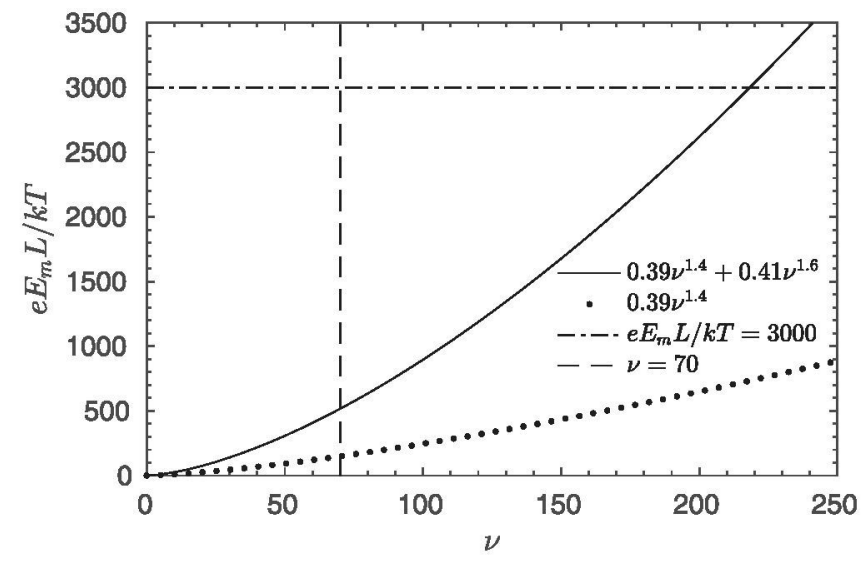

FIG. 3. Operation regimes of thermionic bare tether.

negligible. This is indeed the case. The ratio of ordinates for a dotted-line point and a point directly and well above the solid line gives a length ratio

$$
\frac{\mathrm{BB} * \text { segment length }}{\text { full tether length }} \ll 1,
$$

according to Eq. (15).

\section{CONCLUSIONS}

The present analysis shows that, even without knowing the law of the emitted current within the SCL regime, it is possible to find the parametric domain where SCL emission is ignorable and need not be modelled; that domain is just well above the solid line in Fig. 3. The model proposed involves the value of tether bias at which the transition from SCL to full RD happens, a result that can be taken from the asymptotic kinetic analysis of just cylindrical emissive Langmuir probes. Our analysis further determines the parametric domain where there is no RD emission, which is below that solid line in Fig. 3. Determining that line involved an approximation to SCL emission, which does not affect, however, the well above rule for negligible SCL emission.

The asymptotic analysis was limited to the weak ohmic effects case; moderate ohmic effects are left to later study. Also, the case $R=R_{\max } \approx \lambda_{D e}$ for just OML ion collection was considered; the present study should be expanded by considering any radius, beyond or within OML condition. Furthermore, the influence of different probe-to-electron and ion-to-electron temperature ratios, along with probe radius, on the parametric domain for operational regimes can then be discussed. Broad parametric analyses of the Vlasov-Poisson system for the radial sheath structure can be accomplished, comparing the analytical and numerical ${ }^{21}$ methods.

Finally, the Vlasov-Poisson system solutions should be combined with the longitudinal profile equations to carry out the analysis of tether performance. Of particular interest is comparing TBTs and standard bare tethers, using the hollow cathodes, in such basic tether applications as big debris deorbiting ${ }^{10}$ and generation of artificial auroras. ${ }^{11}$ 


\section{ACKNOWLEDGMENTS}

Work by G. Sánchez-Arriaga was supported by the Ministerio de Economía y Competitividad of Spain (Grant No. RYC-2014-15357).

${ }^{1}$ J. A. Carroll, Acta Astronaut. 13, 165 (1986).

${ }^{2}$ M. D. Grossi, in 2nd International Conference on Tether in Space (Consiglio Nazionale delle Ricerche, Venice/Italy, 1987), pp. 3-8.

${ }^{3}$ M. Cosmo and E. C. Lorenzini, Tethers in Space Handbook, 3rd ed. (NASA CR-97-206807, 1997).

${ }^{4}$ J. R. Sanmartin, M. Martinez-Sanchez, and E. Ahedo, J. Propul. Power 9 , 353 (1993).

${ }^{5}$ J. R. Sanmartin and R. D. Estes, Phys. Plasmas 6, 395 (1999).

${ }^{6}$ S. B. Khan and J. R. Sanmartin, Adv. Space Res. 53, 1370 (2014).

${ }^{7}$ Y. Toda, S. Matsuishi, K. Hayashi, K. Ueda, T. Kamiya, M. Hirano, and H. Hosono, Adv. Mater. 16, 685 (2004).

${ }^{8}$ S. W. Kim and H. Hosono, Philos. Mag. 92, 2596 (2012).

${ }^{9}$ J. D. Williams, J. R. Sanmartin, and L. P. Rand, IEEE Trans. Plasma Sci. 40, 1441 (2012).
${ }^{10}$ J. Sanmartin, A. Sanchez-Torres, S. Khan, G. Sanchez-Arriaga, and M. Charro, Adv. Space Res. 56, 1485 (2015).

${ }^{11}$ J. R. Sanmartin, M. Charro, J. Pelaez, I. Tinao, S. Elaskar, A. Hilgers, and M. Martinez-Sanchez, J. Geophys. Res. A: Space Phys. 111, A11310 (2006).

${ }^{12}$ M. Grossi, in Proceedings of 4th International Conference on Tethers in Space, Science and Technology (Washington DC, 1995).

${ }^{13}$ R. L. Forward, R. P. Hoyt, and C. W. Uphoff, J. Spacecr. Rockets 37, 187 (2000).

${ }^{14}$ G. Sanchez-Arriaga, X. Chen, and E. C. Lorenzini, "Optimal Design and Deorbiting Performance of Thermionic Tethers in Geostationary Transfer Orbits," J. Propul. Power (unpublished).

${ }^{15}$ M. D. Campanell, A. V. Khrabrov, and I. D. Kaganovich, Phys. Rev. Lett. 108, 255001 (2012).

${ }^{16}$ M. D. Campanell and M. V. Umansky, Phys. Rev. Lett. 116, 085003 (2016).

${ }^{17}$ G. L. Delzanno and X. Z. Tang, Phys. Rev. Lett. 113, 035002 (2014).

${ }^{18}$ I. Langmuir and K. B. Blodgett, Phys. Rev. 22, 347 (1923).

${ }^{19} \mathrm{X}$. Chen and J. R. Sanmartin, Phys. Plasmas 19, 073508 (2012).

${ }^{20}$ X. Chen and J. R. Sanmartin, Phys. Plasmas 22, 053504 (2015).

${ }^{21} \mathrm{X}$. Chen and G. Sanchez-Arriaga, "Orbital motion theory and operational regimes for cylindrical probes," Phys. Plasmas (submitted). 